\title{
STRONG MAGNETIC FIELDS IN BIPOLAR OUTFLOWS
}

\author{
M. D. SMITH* ${ }^{*}$ P. W. J. L. BRAND ${ }^{\dagger} \&$ A. MOORHOUSE ${ }^{\ddagger}$, \\ ${ }^{*}$ Dept. of Physics, University of Durham, ${ }^{\dagger}$ Dept. of Astronomy, University of Edinburgh \\ $\mathcal{G} \ddagger$ Dept. of Mathematics, UMIST
}

\begin{abstract}
A supersonic wind from a young star will produce regions of strong magnetic field in the stellar environment. The associated shocks compress the molecular gas, increasing the density $n$, pressure $p$, and field $B$. Crucially, the Alfvén speed, $v_{A} \propto B / n^{1 / 2}$, is also increased since the total shock compression is approximately of the form $\mathrm{B} \propto \mathrm{n}$. But is there any evidence for such high $\mathrm{v}_{A^{-}}$or 'active cloud' - regions within bipolar outflows? We indicate below one implication which has important observable consequences: fast shocks of low Alfvén number $\left(\mathrm{v} / \mathrm{v}_{A}\right)$ now arise. With a low ionization level, the C-shock structure is qualitatively different from the high Alfvén number flows which are common to 'quiescent cloud' conditions. The magnetic-field cushioning now allows molecular hydrogen to survive very fast shocks and broad $\mathrm{H}_{2}$ lines are feasible. We display results which show that the resolved broad lines and line ratio properties in the OMC-1 outflow can be explained with fast bow shocks moving through such active regions.
\end{abstract}

\section{Line profiles from C-shocks}

Shocks in dense molecular clouds are expected to be C-type, in which the low ionized fractions allow ion-magnetosonic waves to propagate upstream, spreading the transition and avoiding the discontinuous jump of a J-shock (see Draine, this volume). This does assume that the ionization level remains low all across the shock - typically limiting the velocity difference between the neutrals and ions/field to $\mathrm{v}_{\text {in }}<40 \mathrm{~km} \mathrm{~s}^{-1}$ (Draine et al 1983, Smith \& Brand 1990a).

We shall mainly look at shocks with $\mathrm{v}>30 \mathrm{~km} \mathrm{~s}^{-1}$ (or curved shocks in which this is satisfied over part of the shock surface) for which line profiles could be resolved by present observations. 'Quiescent' and 'active' cloud regions are here defined by the Alfvén speed. In the former, $\mathrm{v}_{A}<4 \mathrm{~km} \mathrm{~s}^{-1}$. Hence the shock Alfvén number is large in quiescent regions. This implies that the ion/field component possesses only a small fraction of the flow momentum, most of which is in the neutral component. Thus the neutrals are very slowly accelerated and lag way behind the ions. Hence C-shock velocities cannot far exceed 


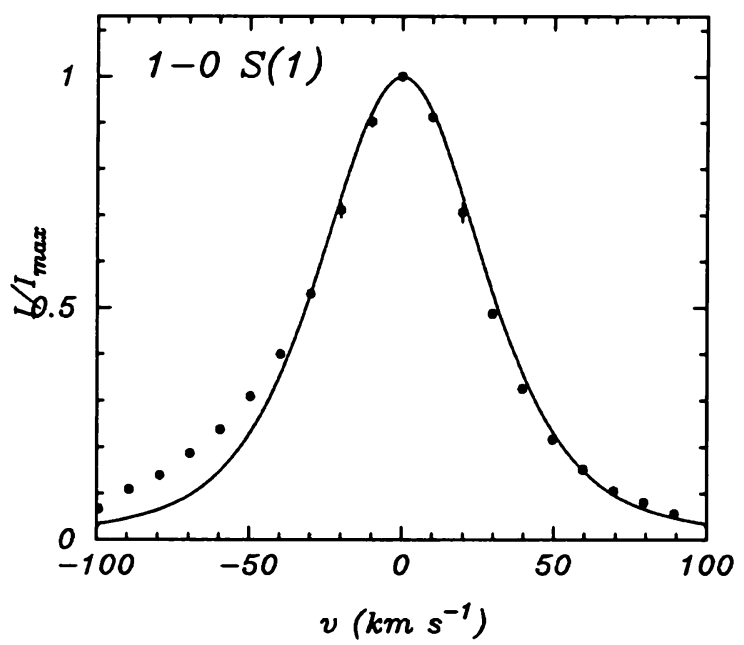

Figure 1: Calculated line profile with the OMC-1 Peak 1 data superimposed.

$\mathrm{v}_{\text {in }}$ and the molecular lines produced are narrow.

In contrast, in active regions $\left(\mathrm{v}_{A}>10 \mathrm{~km} \mathrm{~s}^{-1}\right)$ the low Alfvén number implies that the ions/field contains considerable momentum. The neutrals do not lag far behind the ions - the velocity difference (i.e. the streaming velocity) can be a small fraction of the shock velocity. Hence the shock velocity can be far in excess of the streaming velocity in active regions (Smith et al 1990a,b). Moreover, since the ions heat the neutrals via streaming collisions, the temperature of the molecular hydrogen is tied to the streaming velocity and not the shock velocity. Thus molecular dissociation is similarly inhibited in strong field regions.

We have employed a shock model to determine if the above scenario can provide an explanation for the extremely wide lines observed at peak 1 of OMC-1 ( $\left.\sim 140 \mathrm{~km} \mathrm{~s}^{-1}\right)$. No previous modelling of these lines has proved possible (see Smith \& Brand 1990b). Indeed, we find a reasonable fit without the need of manipulating parameters. Figure 1 displays the data and model for a paraboloidal bow shock of velocity $\mathrm{v}_{w}=250 \mathrm{~km} \mathrm{~s}^{-1}$ moving in the plane of the sky. (The bow calculations take into account the dissociated cap region (bespeckled) and the field direction at each point on the bow surface (figure 2). Each part of the bow is treated as a planar shock element. UV radiation from the leading edge is discussed by Smith \& Brand (1990b).) The magnetic field required is 25 times larger than the 'standard' quiescent value; with $\mathrm{v}_{A}=46.4 \mathrm{~km} \mathrm{~s}^{-1}$ the field is $30 \mu \mathrm{G}$ for an $\mathrm{H}_{2}$ density of $10^{6} \mathrm{~cm}^{-3}$. With an ionized fraction $2.510^{-7}$ the breakdown speed of a planar shock is 130 $\mathrm{km} \mathrm{s}^{-1}$. The $\mathrm{H}_{2} \mathrm{O}$ abundance was set to zero throughout the shock - this value, however, is not crucial to the profiles.

\section{Molecular hydrogen line ratios}

There is now extensive accurate $\mathrm{H}_{2}$ line ratio data for OMC-1. Two factors are apparent: 


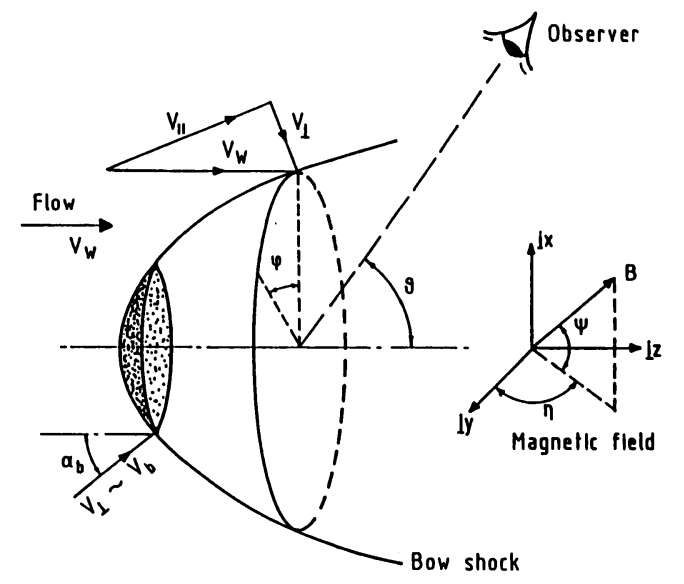

Figure 2: The bow shock.

(1) the ratios cannot be modelled by a constant temperature slab since the excitation temperature increases with the upper energy level of the transitions (Brand et al 1988) and (2) the ratio of $0-0 \mathrm{~S}(13)(17445 \mathrm{~K})$ to $1-00(7)(8365 \mathrm{~K})$ is independent of the position in the outflow as observed by Brand et al (1989a).

We employ the column density ratio (CDR) method to interpret the data. A CDR expresses the column of $\mathrm{H}_{2}$ in a particular energy level to that in the strong 1-0 S(1) line, all the numbers being normalised to that of a $2000 \mathrm{~K}$ slab of size which would reproduce the 1-0 S(1) line intensity. This method is necessary to make accurate quantitative fits in which error bars are of a recognisable size. A column analysis assumes that the $\mathrm{H}_{2}$ is in LTE $\left(\mathrm{n} \geq 10^{6}\right)$ but it remains a useful tool at lower densities.

Remarkably, the bow shock model not only fits the data but possesses a narrow upper envelope (figure 3 ) provided the bow velocity exceeds the breakdown velocity ( $\mathrm{v}>130$ $\mathrm{km} \mathrm{s}^{-1}$ in the example). The CDR envelope is fixed by the shock shape (changing $\mathrm{v}_{w}, \mathrm{~B}$ or $\chi$ shifts the position of the emitting region without changing the ratios), and the cooling function (a high $\mathrm{H}_{2} \mathrm{O}$ production rate leads to quicker downstream cooling and a steeper CDR envelope). A perfect fit is not apparent, with a little too much emission predicted for $\mathrm{T}_{j}$ near $12,000 \mathrm{~K}$, but the scope for detailed modelling is enormous e.g. non-uniform field or distorted shock fronts.

\section{Discussion}

Any 'direct' observation of the magnetic field strength will prove difficult. Evidence through this model may prove irrepressible once $\mathrm{CO}$ and $\mathrm{H}_{2} \mathrm{O}$ line predictions and $\mathrm{H}_{2}$ line mapping is completed. Masers as dense clumps in which the Alfvén speed is large may well be related. Faraday rotation measurements of background extragalactic radio sources limit the ionized fraction rather than the field. Ion-neutral line profile differences are measurable only when 


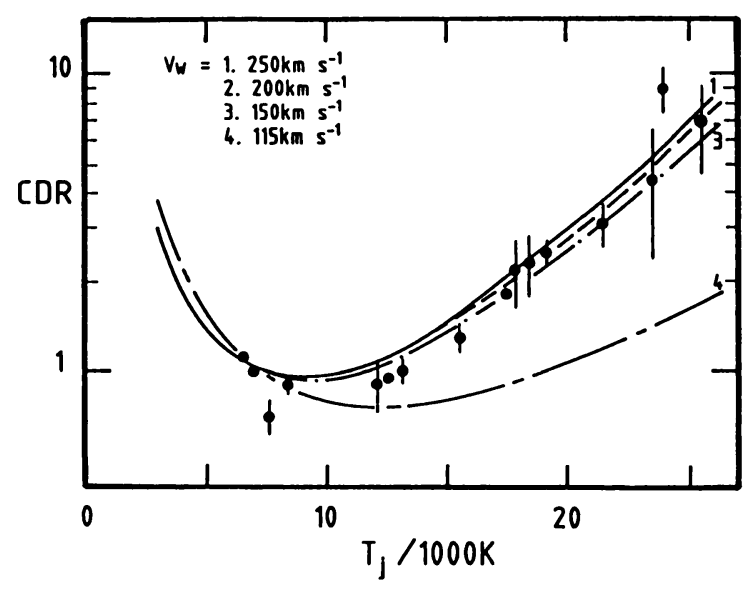

Figure 3: Line ratios expressed as column density ratios for the strong field example compared to OMC-1 Peak 1 data.

high ionization levels occur. High fields cannot be inferred to hold upo the active cloud such reasoning applies only to quiescent cloud regions.

Two-component outflows are predicted by this 'shock absorber' model. One outflow produces a region with $\mathrm{v}_{A} \sim 10-60 \mathrm{~km} \mathrm{~s}^{-1}$ - hence tentatively related to the CO bipolar outflows. The second high-velocity system drives through this medium. The speeds of 80 $300 \mathrm{~km} \mathrm{~s}^{-1}$ correspond to $\mathrm{HH}$ objects and jets.

A J-shock model can reproduce the line ratio data in OMC-1 with the pressure as the one modelling parameter. A low $\mathrm{H}_{2} \mathrm{O}$ abundance is required. Also a large number of preaccelerated clumps or high-velocity turbulence is postulated in an attempt to explain the line widths (Brand et al 1989b). The pre-acceleration (after which $\mathrm{H}_{2}$ must reform) is especially difficult to achieve within Peak 1 - a strong peak which subtends a small angle with the distant outflow centre.

\section{References}

Brand, P.W.J.L., Moorhouse, A., Burton, M.G., Geballe, T.R., Bird, M., \& Wade, R., 1988. Astrophys. J. Letters 334, L103.

Brand, P., Toner. M.P. et al. 1989a. M.N.R.A.S. 236, 929.

Brand, P., Toner. M.P. et al. 1989b. M.N.R.A.S. 237, 1009.

Draine, B.T., Roberge, W.G. \& Dalgarno, A., 1983. Ap. J., 264, 485.

Smith, M.D. \& Brand, P.W.J.L., 1990a. M.N.R.A.S. 242, 495.

Smith, M.D. \& Brand, P.W.J.L., 1990b. M.N.R.A.S. 245, 108.

Smith, M.D., Brand, P.W.J.L. \& Moorhouse, A., 1990a \& 1990b. M.N.R.A.S. submitted 